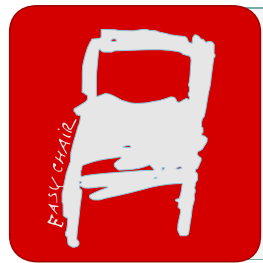

EPiC Series in Health Sciences

Volume 3, 2019, Pages 311-314

CAOS 2019. The 19th Annual Meeting of the International Society for Computer Assisted Orthopaedic Surgery

\title{
Can standard implants reproduce the native kinematics of a TKA patient?
}

\author{
Maria Paz Quilez ${ }^{1}$, Hendrik Delport ${ }^{2}$, Roel Wirix-Speetjens ${ }^{1}$, Mariska \\ Wesseling ${ }^{1}$, Maria Angeles Perez Anson ${ }^{3}$, Ilse Jonkers ${ }^{2}$, Jos Vander Sloten ${ }^{2}$ \\ ${ }^{1}$ Materialise NV, Leuven, Belgium \\ ${ }^{2}$ Katholieke Universitet Leuven, Belgium \\ ${ }^{3}$ University of Zaragoza, Spain \\ mariapaz .quilez@materialise.be
}

\begin{abstract}
Total knee arthroplasty (TKA) is a reliable surgical procedure, yet up to a fifth of primary implant patients remains unsatisfied. Musculoskeletal modeling (MSM) has the potential to explore the relationship between implant alignment and functional outcome [3]. Consequently, implant alignment can be quantitatively optimized to restore the preTKA joint behavior and, therefore, achieve the most favorable functional outcome for the specific patient. For this reason, we developed a method to optimize the implant alignment, with the aim of restoring the native kinematics and ligament elongations of the patient before undergoing TKA. Subject-specific optimization towards ligament elongations demonstrated to accurately emulate the pre-TKA ligament behavior, in contrast to the mechanically aligned approach. However, the values of the optimized implant positions resulting from the pre-TKA kinematic optimization were extreme in some cases. The presented modelling approach is a promising starting point for allowing surgeons to evaluate the patient-specific implant alignment and restore the patientspecific biomechanics.
\end{abstract}

\section{Introduction}

Total knee arthroplasty (TKA) is a reliable surgical procedure, yet up to a fifth of primary implant patients remains unsatisfied. One of the most common procedures in TKA focuses on achieving good alignment of the mechanical axes, which not always results in the desired functional outcome for the patient. Therefore, new approaches as soft-tissue balancing [1] and kinematics alignment [2] have been emerging. Although pre-operative planning tools were recently introduced to support the surgeon in obtaining mechanical alignment, these planning tools do not support soft-tissue balancing and kinematic alignment approaches yet. 
Musculoskeletal modeling (MSM) has the potential to explore the relationship between implant alignment and functional outcome [3]. Consequently, implant alignment can be quantitatively optimized to restore the pre-TKA joint behavior and, therefore, achieve the most favorable functional outcome for the specific patient. For this reason, we developed a method to optimize the implant alignment, with the aim of restoring the native kinematics and ligament elongations of the patient before undergoing TKA.

\section{Method}

A patient-specific musculoskeletal model (MSM) was previously developed and validated by Vanheule et al [3]. This model predicts kinematics and ligament length elongations when squatting, for both pre-TKA and post-TKA knees. In this study, we used the model and the four cadaveric experiments performed in that work.

Pre-TKA tibio-femoral kinematics and medial and lateral collateral ligament (MCL and LCL) elongations were computed for the four specimens using the pre-TKA knee model. Next, the post-TKA model using a posterior-stabilized (PS) implant system was used to calculate the kinematics and ligament elongations for a combination of different femoral and tibial implant positions. Implant positions were determined by the following parameters: varus-valgus (VV), proximal-distal (PD), slope (Sl), internal-external (IE) for both femoral and tibial component, and also anterior-posterior (AP) for the femoral component. 130 simulations were run based on a design of experiments (DOE), varying each variable from $-5 \mathrm{~mm}$ or $-5^{\circ}$ to $+5 \mathrm{~mm}$ or $+5^{\circ}$.

The results of the kinematics and ligament elongations obtained from the simulations were used to determine the optimal implant position using a response surface method (RSM). The optimal implant positions were defined as the positions with the smallest error between the pre-TKA and the post-TKA tibio-femoral kinematics and ligament elongations. Then, those results were compared with the results of the mechanical alignment technique.

\section{Results}

The optimal set of implant position parameters that produced pre-TKA ligament elongations were calculated for the four specimens (Figure 1a). The obtained optimized positions were used to compute the ligament elongations for each specimen, and compared to the mechanically aligned approach. This comparison revealed a meaningful difference between the optimized and mechanically aligned positions (Figure 2). 
Can standard implants reproduce the native kinematics of a TKA patient?

M. P. Quilez et al.

\begin{tabular}{|c|c|c|c|c|c|c|}
\hline & $\mathrm{VV}_{\mathrm{fem}}$ & \multicolumn{2}{|c|}{$\mathrm{PD}_{\mathrm{fem}}$} & $\mathrm{Sl}_{\mathrm{fem}}$ & $\mathrm{IE}_{\mathrm{fem}}$ & $\mathrm{AP}_{\mathrm{fem}}$ \\
\hline Spec 1 & $1.3^{\circ}$ var & or $\quad 0.6 \mathrm{~m}$ & $\mathrm{~m}$ dis & $0.2^{\circ} \mathrm{po}$ & os $2.0^{\circ} \mathrm{int}$ & $0.6 \mathrm{~mm}$ ant \\
\hline Spec 2 & $1.1^{\circ} \mathrm{val}$ & $1.7 \mathrm{~m}$ & m prox & $0.1^{\circ} \mathrm{po}$ & os $0.1^{\circ}$ int & $0.7 \mathrm{~mm}$ ant \\
\hline Spec 3 & $3.1^{\circ} \mathrm{val}$ & l $2.6 \mathrm{~m}$ & $\mathrm{~m}$ prox & $0.2^{\circ} \mathrm{po}$ & os $1.9^{\circ} \mathrm{ext}$ & t $\quad 1.1 \mathrm{~mm}$ pos \\
\hline Spec 4 & $1.7^{\circ} \mathrm{var}$ & or $\quad 0.4 \mathrm{~m}$ & $\mathrm{~m}$ dis & $0.4^{\circ} \mathrm{po}$ & s $1.5^{\circ}$ ext & $1.8 \mathrm{~mm}$ pos \\
\hline \multicolumn{7}{|c|}{ Femoral component } \\
\hline & & $V V_{\text {tib }}$ & \multicolumn{2}{|l|}{$\mathrm{PD}_{\mathrm{tib}}$} & $\mathrm{Sl}_{\mathrm{tib}}$ & $\mathrm{IE}_{\mathrm{tib}}$ \\
\hline & Spec 1 & $1.3^{\circ}$ var & \multicolumn{2}{|c|}{$1.6 \mathrm{~mm}$ dis } & $0.1^{\circ}$ ant & $0.2^{\circ}$ ext \\
\hline & Spec 2 & \multirow{2}{*}{$\begin{array}{l}1.5^{\circ} \text { val } \\
2.5^{\circ} \text { val }\end{array}$} & \multicolumn{2}{|c|}{$4.5 \mathrm{~mm}$ dis } & $0.7^{\circ}$ pos & $0.1^{\circ} \mathrm{ext}$ \\
\hline & Spec 3 & & \multicolumn{2}{|c|}{$2.9 \mathrm{~mm}$ dis } & $0.1^{\circ}$ pos & \\
\hline & Spec 4 & $2.0^{\circ}$ var & \multicolumn{2}{|c|}{$0.2 \mathrm{~mm}$ prox } & $0.1^{\circ} \operatorname{pos}$ & $\begin{array}{l}0.5^{\circ} \text { ext } \\
0.2^{\circ} \text { int }\end{array}$ \\
\hline
\end{tabular}

a) Optimization towards ligament elongations

\begin{tabular}{|c|c|c|c|c|c|c|c|}
\hline & $\mathrm{VV}_{\mathrm{fem}}$ & \multicolumn{2}{|c|}{$\mathrm{PD}_{\mathrm{fem}}$} & \multicolumn{2}{|c|}{$\mathrm{Sl}_{\mathrm{fem}}$} & $\mathrm{IE}_{\mathrm{fem}}$ & $\mathrm{AP}_{\text {fem }}$ \\
\hline Spec 1 & $2.3^{\circ} \mathrm{var}$ & \multicolumn{2}{|c|}{$3.7 \mathrm{~mm}$ dis } & \multicolumn{2}{|c|}{$5.2^{\circ}$ pos } & $0.9^{\circ}$ int & $6.5 \mathrm{~mm}$ pos \\
\hline Spec 2 & $2.5^{\circ} \mathrm{var}$ & \multicolumn{2}{|c|}{$1.2 \mathrm{~mm}$ prox } & \multicolumn{2}{|c|}{$7.2^{\circ}$ ant } & $22^{\circ} \mathrm{ext}$ & ext $\quad 0.5 \mathrm{~mm}$ pos \\
\hline Spec 3 & $2.1^{\circ} \mathrm{var}$ & & & $0.6^{\circ}$ int & $6.2 \mathrm{~mm}$ pos \\
\hline Spec 4 & $2.1^{\circ}$ var & \multicolumn{2}{|c|}{$\begin{array}{l}0.2 \mathrm{~mm} \text { dis } \\
4.4 \mathrm{~mm} \text { dis }\end{array}$} & \multicolumn{2}{|c|}{$\begin{array}{l}7.0^{\circ} \mathrm{pos} \\
4.3^{\circ} \mathrm{pos}\end{array}$} & $\begin{array}{l}0.6^{\circ} \text { int } \\
3.3^{\circ} \text { int }\end{array}$ & $3.6 \mathrm{~mm}$ pos \\
\hline \multicolumn{8}{|c|}{ Femoral component } \\
\hline & & $\mathrm{VV}_{\text {tib }}$ & \multicolumn{2}{|l|}{$\mathrm{PD}_{\text {tib }}$} & \multicolumn{2}{|l|}{$\mathrm{Sl}_{\mathrm{tib}}$} & $\mathrm{IE}_{\mathrm{tib}}$ \\
\hline & Spec 1 & $0.1^{\circ} \mathrm{val}$ & \multicolumn{2}{|c|}{$4.5 \mathrm{~mm}$ dis } & \multicolumn{2}{|c|}{$15.2^{\circ} \mathrm{pos}$} & $0.6^{\circ}$ ext \\
\hline & Spec 2 & $3.9^{\circ} \mathrm{val}$ & \multicolumn{2}{|c|}{$4.6 \mathrm{~mm}$ dis } & & $12.6^{\circ} \mathrm{ext}$ \\
\hline & Spec 3 & $4.5^{\circ} \mathrm{val}$ & \multicolumn{2}{|c|}{$\begin{array}{l}4.6 \mathrm{~mm} \text { dis } \\
7.5 \mathrm{~mm} \text { dis }\end{array}$} & \multicolumn{2}{|c|}{$0.0^{\circ}$ pos } & $\begin{array}{l}12.6^{\circ} \mathrm{ext} \\
7.3^{\circ} \mathrm{ext}\end{array}$ \\
\hline & Spec 4 & $5.7^{\circ} \mathrm{var}$ & \multicolumn{2}{|c|}{$2.6 \mathrm{~mm}$ dis } & \multicolumn{2}{|c|}{$9.2^{\circ}$ pos } & $4.4^{\circ}$ ext \\
\hline
\end{tabular}

b) Optimization towards kinematics

Figure 1. Optimization of the implant positions towards the pre-TKA situation
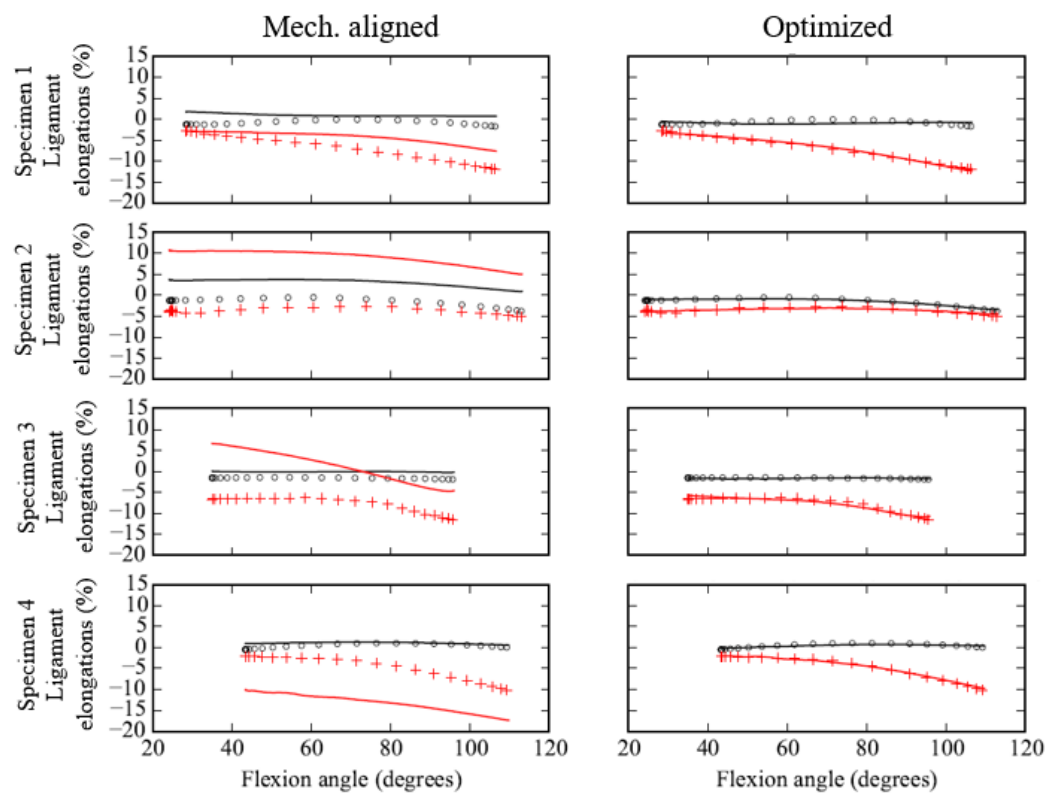

\begin{tabular}{|ll|}
\hline Pre-TKA MCL & - Post-TKA MCL \\
+ & + Pre-TKA LCL
\end{tabular}

Figure 2. Comparison of mechanically aligned and optimized ligament elongations. Pre-TKA (circles and crosses) and mechanically aligned/optimized (line) for MCL (black) and LCL (red) length changes are shown.

The implant position was also optimized towards the pre-TKA kinematics (Figure 1b). Interestingly, these optimal solutions were associated with high values of tibial slopes for specimen $1\left(15.2^{\circ}\right), 3\left(8.9^{\circ}\right)$ and $4\left(9.2^{\circ}\right)$ 


\section{Discussion}

Asseln et al. [4] evaluated the kinematics of the knee joint and its relevance for TKA implant design. Similarly, Twiggs et al. [5] reported correlations between kinematics and patient satisfaction. However, to our knowledge there is no study about the use of MSM for directly supporting the surgeon decisiontaking.

As shown in figure 2, subject-specific optimization towards ligament elongations demonstrated to accurately emulate the pre-TKA ligament behavior, in contrast to the mechanically aligned approach.

The values of the optimized implant positions resulting from the pre-TKA kinematic optimization were extreme in some cases. We attribute this to the PS mechanism that restricts mainly the motion in the anterior-posterior and internal-external directions. Assigning lower weight to those parameters in the optimization process would be more representative of the PS system and, therefore, it could likely give more realistic results. Additionally as future work, a less constrained implant system as a cruciateretaining (CR) implant will be evaluated, as it could provide more freedom to the optimization process and would allow approximating more closely the native knee behavior.

An important limitation of this study is that the experimental data is limited to four cadaveric specimens and results need to be confirmed in a larger dataset.

We conclude that the presented modelling approach is a promising candidate for allowing surgeons to evaluate the patient-specific implant alignment and restore the patient-specific biomechanics. The current method provides a solid starting point to enhance the integration of computational models into clinical practice and evolve towards a patient-specific optimized approach.

\section{References}

[1] U. W. Tobias Wyss, Andreas J. Schuster, Bernhard Christen, "Tension controlled ligament balanced total knee arthroplasty : 5-year results of a soft tissue orientated surgical technique," Arch. Orthop. Trauma Surg., vol. 128, no. 2, pp. 129-135, 2008.

[2] Y. S. Lee et al., "Kinematic alignment is a possible alternative to mechanical alignment in total knee arthroplasty," Knee Surgery, Sport. Traumatol. Arthrosc., vol. 25, no. 11, pp. 3467-3479, 2017.

[3] V. Vanheule et al., "Evaluation of predicted knee function for component malrotation in total knee arthroplasty,” Med. Eng. Phys., vol. 40, pp. 56-64, 2017.

[4] M. Asseln, L. Berger, M. Verjans, and K. Radermacher, "Effects of the medial and lateral tibial slope on knee joint kinematics in total knee arthroplasty," Curr. Dir. Biomed. Eng., vol. 4, no. 1, pp. 207-211, 2018.

[5] P. Joshua Twiggs, BE, Edgar Wakelin, PhD, Justin Roe, MD, David Dickison, MD, Brett Fritsch, MD, Brad Miles, PhD, Andrew Ruys, "Patient Specific Simulated Dynamics following TKA Correlate with Patient Reported Outcomes," J. Arthroplasty, vol. 33, no. 9, pp. 2843-2850, 2018. 